Research Paper

\title{
Non-invasive Analysis of Genomic Copy Number Variation in Patients with Hepatocellular Carcinoma by Next Generation DNA Sequencing
}

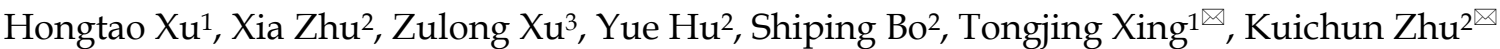 \\ 1. Department of Infectious Diseases, Taizhou People's Hospital, Taizhou, Jiangsu, China. \\ 2. Yikon Genomics Inc. Taizhou, Jiangsu, China. \\ 3. Department of Laboratory Medicine, Taizhou People's Hospital, Taizhou, Jiangsu, China.
}

$\triangle$ Corresponding author: kzhu68@hotmail.com; xingtj518@sina.com

() 2015 Ivyspring International Publisher. Reproduction is permitted for personal, noncommercial use, provided that the article is in whole, unmodified, and properly cited. See http://ivyspring.com/terms for terms and conditions.

Received: 2014.10.07; Accepted: 2014.12.09; Published: 2015.01.18

\begin{abstract}
To explore new molecular diagnosis approaches for early detection and differential diagnosis of hepatocellular carcinoma (HCC), we analyzed genomic copy number variations (CNV) using plasma cell-free DNA from patients with HCC by next generation DNA sequencing. Plasma samples from 31 patients with $\mathrm{HCC}$ and 8 patients with chronic hepatitis or cirrhosis were analyzed. In HCC group, most samples with large tumor size (tumor dimension greater than $50 \mathrm{~mm}$ ) showed CNVs that are visually recognizable at chromosome CNV plots, few samples with small tumor and none samples with chronic liver diseases showed CNVs recognizable at CNV plots. CNV Z score analysis showed significant CNVs in samples with HCC and chronic liver diseases although more significant changes were found in $\mathrm{HCC}$ group, some are differentially valuable (such as gain in $1 q, 7 q$, and $19 q$ in $\mathrm{HCC}$ ), while others are less differentially valuable (such as loss in $4 q$, 13q, gain in 17q, 22q). We proposed a CNV scoring method that generated positive result in 26 of the $31 \mathrm{HCC}$ patients $(83.9 \%$ ) or 11 of the $16 \mathrm{HCC}$ with tumor dimension $50 \mathrm{~mm}$ or less $(68.8 \%)$ or 4 of the $7 \mathrm{HCC}$ with tumor dimension $30 \mathrm{~mm}$ or less $(57.1 \%)$, while all the 8 samples with chronic hepatitis or cirrhosis scored negative. Ten HCC patients had normal or low serum AFP levels, among them, 7 were scored positive by CNV analysis, including 4 with tumor dimension 50 $\mathrm{mm}$ or less. Our study suggested that non-invasive genomic CNV analysis using plasma samples could be a valuable tool for early detection and differential diagnosis of HCC. Although CNV analysis itself cannot establish the diagnosis, it can help identify patients at high risk for $\mathrm{HCC}$ among patients with chronic liver diseases, which would prompt closer and more frequent surveillance for early tumor detection and intervention.
\end{abstract}

Key words: Genomic Copy Number Variation, HCC, DNA sequencing

\section{Introduction}

According to WHO GLOBOCAN 2012 statistics, liver cancer is the fifth most common cancer type in men, and the second most common cause of death from cancer worldwide. Hepatocellular carcinoma (HCC) develops often with underlying chronic liver diseases such as chronic hepatitis $(\mathrm{CH})$ or cirrhosis (CR). Most patients were diagnosed at late stages, having a survival time often less than 6 months after initial diagnosis. However, the 5-year survival rate for patients with early HCC after resection ranged from $27 \%$ to $81 \%$ [1]. Early detection and differential diagnosis is the key to the success of tumor resection and good prognosis. Current HCC screening methods include mainly serum AFP test, computed tomogra- 
phy scan and ultrasonography examination.

In the past several years, DNA sequencing technology has evolved dramatically. Next generation DNA sequencing (NGS) has brought genome sequencing to clinical laboratories. The huge reduction in the sequencing cost and increase in sequencing efficiency, the incomparable sequencing throughput, sensitivity, and accuracy all make NGS the most promising laboratory technology for cancer genomics, cancer genetics, early diagnosis and personalized treatment of cancer patients in next decade [2-4]. Combined with the superior NGS technology, non-invasive diagnosis of cancers using plasma cell-free DNA (cfDNA) from cancer patients has proven to be feasible. The use of cfDNA circumvents biopsy or surgery, but still being able to get relatively representative and complete genomic and genetic information of cancer cells [5-9].

Cancer is a genetic disorder. Advanced cancer cells carry an array of genetic variants and genomic abnormalities, mutations, insertions, deletions, translocations, inversions, amplification are some of the most common genetic and genomic changes in cancer cells. Deletion and amplification can be identified by copy number variation (CNV) analysis. Different kinds of CNVs have been reported in HCC, mostly by comparative genomic hybridization analysis with genetic materials from tumor tissues. The most common CNVs reported in HCC include gain in $1 \mathrm{q}, 8 \mathrm{q}$, $17 q, 20 q$, and loss in 4q, 13q, 14q, 16q,1p, 8p, 9p, 17p [10-13].

We aimed to screen for genomic CNVs using plasma cfDNA from HCC patients by NGS technology, and such CNVs might help for early detection or differential diagnosis of HCC in the context of underlying chronic liver diseases. After thorough analysis of the CNV data, we proposed a CNV scoring method that showed good performance in our study and with our set of clinical data.

\section{Materials and Methods}

\section{Patients}

All patients involved in the study were recruited at local hospital for diagnosis and treatment of liver diseases. Diagnosis of chronic hepatitis $(\mathrm{CH})$, cirrhosis (CR), and hepatocellular carcinoma (HCC) were made following guidelines from Society of Hepatology, Chinese Medical Association, and blood samples were collected at the diagnosis of the diseases. Measurement of tumor dimension was performed by computed tomography scan. Patients with single tumor or nodule were included in the study. Serum AFP test was performed with chemiluminescence method with reference value less than $10 \mathrm{ng} / \mathrm{ml}$, HBV DNA copy number was measured with fluorescence qPCR method, both were performed using commercial kits approved for use in clinical laboratories by the Ministry of Health. Blood samples were drawn into tubes with EDTA as anti-coagulant. Plasma was prepared by centrifuging blood samples at $2000 \mathrm{~g}$ for 5 mins, stored in aliquots at $-80^{\circ} \mathrm{C}$, and sent for test within 3 months.

The use of human blood samples for clinical research was performed following Institutional Review Board requirements and with written informed consent from patients. The study was approved by the Ethical and Scientific Committees of Taizhou People's Hospital.

\section{Cell-free DNA isolation}

Cell-free DNA (cfDNA) was extracted from plasma using BloodGen mini kit (CoWin Inc, Beijing, China) following manufacturer's protocol.

\section{Library construction, sequencing, and general data processing}

Sequencing library construction, QC, NGS sequencing, general data processing were performed as described previously [14]. For CNV analysis, Illumina flowcells (single read length $40 \mathrm{bp}$ ) were used for sequencing, with 10-15 $\mathrm{M}$ reads each sample and targeted sequencing depth at 0.1-0.2 $\mathrm{x}$.

\section{CNV analysis}

Mapped reads were normalized to relative reads number at $1000 / 150 \mathrm{~kb}$ per bin after GC correction. Relative reads number in each bin was compared with that in the control group to calculate CNV. CNV Z score analysis with Z-test was performed as described previously [9]. CNV control consists of data from 259 healthy subjects. CNV analysis was performed using a set of proprietary Perl scripts, CNV visualization was processed with a proprietary $\mathrm{R}$ script. In this study, $150 \mathrm{~kb}$ per bin data were used for chromosome CNV plots, and $1 \mathrm{M}$ bp per bin data were used for CNV Z score analysis, and only data from autosomes were included in CNV Z score analysis.

Major CNVs identified were verified by fluorescence qPCR. One representative HCC sample and one CR1 sample were chosen for the purpose of verification. qPCR target regions were chosen based on the $\mathrm{CNV} \mathrm{Z}$ score analysis results, one bin for each chromosome arm was chosen as amplification target region, and two sets of qPCR primers were designed from two different regions in the bin chosen. The two housekeeping genes ( $\beta$-actin and $\beta$-tubulin) were used as internal controls for the qPCR. The qPCR was performed using UltraSYBR qPCR Mix (CoWin Inc, Beijing, China), 4 ng cfDNA template per reaction, and 
Roche LightCycle 480 System. The Ct values of housekeeping genes were used to normalize input DNA between samples. Ct values of target regions in each sample were compared with $\mathrm{Ct}$ values of housekeeping genes of the same sample for estimation of CNVs. Primer sequences were shown in Table A.

Table A. Primer sequences used in qPCR analysis.

\begin{tabular}{ll}
\hline Primer name & Primer sequence \\
\hline $1 \mathrm{qaF}$ & CAGATTTGGATGCGCTTTTT \\
$1 \mathrm{qbF}$ & GAAGCCTCACAATCACAGCA \\
$7 \mathrm{qaF}$ & AAGTGACGATTTTGGCCCAC \\
$7 \mathrm{qbF}$ & TATCTCCGGGCCACAACTTT \\
$19 \mathrm{qaF}$ & GGGAAGGAACAGCAGCTCTA \\
$19 \mathrm{qbF}$ & TTGCAACACTACACACCAGC \\
$1 \mathrm{qaR}$ & CTGGAACGCTACGAGGATGT \\
$1 \mathrm{qbR}$ & AACCCCTTCCACTTGGTTCT \\
$7 \mathrm{qaR}$ & AAGCCAAGGATTCGTACCCA \\
$7 \mathrm{qbR}$ & ACGAGAATTGCTTGAACCCG \\
$19 \mathrm{qaR}$ & TGGGAGCCATTGAAGCATTT \\
$19 \mathrm{qbR}$ & TGTGCAGCATAGTGAGACCA \\
actinF & TACACCCCCACTCAAGGGA \\
actinR & CTCCCCAGGTACCCCAGTTT \\
tubulinF & CACCAAGGAAGTGGACCAGCAAC \\
tubulinR & ATGTCCATCCCTTCGCTGGTGTA \\
\hline
\end{tabular}

\section{Results}

\section{Patients and plasma cfDNA}

Thirty one patients with HCC were included in the study. Patient information was summarized in Table 1. Seven patients had tumor size no more than $30 \mathrm{~mm}, 16$ patients had tumor size no more than 50 $\mathrm{mm}$. Serum AFP level was elevated to different extents in 26 patients. All but two had HBV infection.

cfDNA was successfully extracted using a commercial kit from all the 31 cancer plasma samples. Plasma cfDNA from most cancer patients was found in the range of $5-50 \mathrm{ng} / \mathrm{ml}$, with a few patients with large tumor size having plasma cfDNA over 100 $\mathrm{ng} / \mathrm{ml}$ (Table 2). Blood samples with hemolysis were excluded. The plasma cfDNA concentration showed poor correlation with tumor size in general (Fig. 1A). If patients were divided into three groups, with tumor size $30 \mathrm{~mm}$ or less, 31-60 mm, greater than $60 \mathrm{~mm}$, it was found that plasma cfDNA concentration steadily increased with the increase of tumor size (Fig.1B).

Eight patients with $\mathrm{CH}$ or $\mathrm{CR}$ were also included in the study as controls. Plasma cfDNA from chronic liver diseases was found in the range of $3-25 \mathrm{ng} / \mathrm{ml}$ (Table 3).

\section{Chromosome CNV analysis}

The mapped reads from NGS were processed through GC correction, normalization, and comparison with the reads in the CNV control group in each window (bin) for chromosome CNV ratio calculation. Visual CNV change was defined as visible intense segmental increase or decrease in the $\mathrm{CNV}$ ratio in the $150 \mathrm{~kb}$ per bin CNV plots. Scattered dots above or below the baseline were not counted. Read breaks due to repeat sequences (close to centromere or telomere) that appear in every sample were ignored (such as in the middle of Chr 1 and Chr 9 in Figure 2). Among 31 samples from HCC patients, 13 showed visible CNV changes, including 4 with tumor size $50 \mathrm{~mm}$ or less, and 1 with tumor size $30 \mathrm{~mm}$ or less. None of the $\mathrm{CH}$ or CR samples showed CNV changes that can be recognized at $150 \mathrm{~kb}$ per bin CNV plots (Figure 2). Common CNVs observed include gain in $1 \mathrm{q}, 7 \mathrm{q}, 17 \mathrm{q}$, $19 q, 20 q$, and loss in 1p, 4q, 13q, 17p. Additional visual CNV change was summarized in Supplementary Material: Figure S1.

Table 1. Summary of clinical information of HCC patients

\begin{tabular}{|c|c|c|c|c|c|c|}
\hline patient ID & age & gender & $\begin{array}{l}\text { tumor size } \\
\text { (length, } \\
\mathrm{mm} \text { ) }\end{array}$ & $\begin{array}{l}\text { tumor size } \\
\text { (width, } \\
\text { mm) }\end{array}$ & $\begin{array}{l}\text { Serum } \\
\text { AFPa }\end{array}$ & $\begin{array}{l}\text { Serum } \\
\text { HBV } \\
\text { DNA }^{b}\end{array}$ \\
\hline HCC1 & 56 & $\mathrm{~F}$ & 19 & 18 & $\mathrm{M}$ & $\mathrm{L}$ \\
\hline HCC2 & 65 & $\mathrm{~F}$ & 22 & 17 & $\mathrm{~L}$ & $\mathrm{~L}$ \\
\hline HCC3 & 27 & $\mathrm{M}$ & 41 & 28 & $\mathrm{H}$ & $\mathrm{L}$ \\
\hline HCC4 & 62 & $\mathrm{M}$ & 31 & 27 & $\mathrm{~L}$ & $\mathrm{H}$ \\
\hline HCC5 & 59 & M & 30 & 30 & $\mathrm{~N}$ & $\mathrm{~L}$ \\
\hline HCC6 & 55 & $\mathrm{M}$ & 19 & 17 & $\mathrm{M}$ & $\mathrm{L}$ \\
\hline HCC7 & 57 & $\mathrm{M}$ & 35 & 29 & $\mathrm{~N}$ & $\mathrm{~L}$ \\
\hline HCC8 & 55 & $\mathrm{M}$ & 37 & 32 & $\mathrm{H}$ & $\mathrm{L}$ \\
\hline HCC9 & 53 & M & 35 & 26 & $\mathrm{~N}$ & $\mathrm{~N}$ \\
\hline HCC10 & 43 & $\mathrm{M}$ & 20 & 18 & $\mathrm{~N}$ & $\mathrm{H}$ \\
\hline HCC11 & 65 & M & 48 & 40 & $\mathrm{H}$ & $\mathrm{N}$ \\
\hline HCC12 & 54 & $\mathrm{M}$ & 16 & 14 & $\mathrm{~N}$ & $\mathrm{~L}$ \\
\hline HCC13 & 49 & $\mathrm{~F}$ & 36 & 30 & $\mathrm{H}$ & $\mathrm{M}$ \\
\hline HCC14 & 29 & M & 45 & 28 & $\mathrm{H}$ & $\mathrm{L}$ \\
\hline HCC15 & 64 & $\mathrm{~F}$ & 35 & 33 & $\mathrm{H}$ & $\mathrm{H}$ \\
\hline HCC16 & 32 & M & 65 & 58 & $\mathrm{H}$ & $\mathrm{L}$ \\
\hline HCC17 & 49 & $\mathrm{M}$ & 88 & 60 & $\mathrm{M}$ & $\mathrm{M}$ \\
\hline HCC19 & 49 & M & 88 & 52 & $\mathrm{H}$ & M \\
\hline HCC20 & 76 & $\mathrm{M}$ & 75 & 58 & $\mathrm{M}$ & $\mathrm{L}$ \\
\hline HCC21 & 71 & $\mathrm{M}$ & 96 & 89 & $\mathrm{H}$ & $\mathrm{H}$ \\
\hline HCC23 & 60 & $\mathrm{M}$ & 99 & 83 & $\mathrm{H}$ & $\mathrm{L}$ \\
\hline HCC24 & 46 & $\mathrm{M}$ & 93 & 70 & $\mathrm{H}$ & $\mathrm{L}$ \\
\hline HCC26 & 47 & $\mathrm{M}$ & 60 & 50 & $\mathrm{~L}$ & $\mathrm{H}$ \\
\hline HCC27 & 56 & $\mathrm{M}$ & 56 & 54 & $\mathrm{~N}$ & $\mathrm{~L}$ \\
\hline HCC28 & 44 & $\mathrm{M}$ & 78 & 49 & $\mathrm{H}$ & $\mathrm{L}$ \\
\hline HCC29 & 80 & $\mathrm{~F}$ & 65 & 36 & $\mathrm{H}$ & $\mathrm{L}$ \\
\hline НСС30 & 40 & M & 119 & 106 & $\mathrm{H}$ & $\mathrm{M}$ \\
\hline HCC29a & 57 & $\mathrm{M}$ & 90 & 81 & $\mathrm{~N}$ & $\mathrm{M}$ \\
\hline HCC $29 b$ & 53 & M & 95 & 60 & $\mathrm{H}$ & $\mathrm{L}$ \\
\hline $\mathrm{HCC} 29 \mathrm{c}$ & 73 & $\mathrm{~F}$ & 114 & 86 & $\mathrm{H}$ & $\mathrm{H}$ \\
\hline HCC5a & 59 & M & 28 & 21 & $\mathrm{H}$ & $\mathrm{H}$ \\
\hline
\end{tabular}


Table 2. CNV analysis of cfDNA samples from patients with $\mathrm{HCC}$

\begin{tabular}{|c|c|c|c|c|c|c|c|c|c|c|}
\hline $\begin{array}{l}\text { patient } \\
\text { ID }\end{array}$ & $\begin{array}{l}\text { cfDNA } \\
(\mathrm{ng} / \mathrm{ml})\end{array}$ & AFP & $\begin{array}{l}\text { CNV } \\
\text { plot } \\
\text { change }\end{array}$ & $1 q^{+}$ & $7 q^{+}$ & $19 q^{+}$ & $1 p-$ & $9 q-$ & $14 \mathrm{q}-$ & $\begin{array}{l}\mathrm{CNV} \\
\text { score }^{\mathrm{a}}\end{array}$ \\
\hline HCC1 & 8.36 & $\mathrm{M}$ & - & - & - & - & - & + & - & 0.5 \\
\hline HCC2 & 42.39 & $\mathrm{~L}$ & - & - & + & - & - & + & + & $\underline{3}$ \\
\hline HCC3 & 27.10 & $\mathrm{H}$ & - & - & - & + & - & - & - & 2 \\
\hline HCC4 & 33.83 & $\mathrm{~L}$ & - & + & - & - & - & - & + & $\underline{2.5}$ \\
\hline HCC5 & 7.03 & $\mathrm{~N}$ & - & - & - & - & - & - & + & $\underline{0.5}$ \\
\hline HCC6 & 21.00 & M & - & - & - & + & + & + & - & 3 \\
\hline HCC7 & 70.32 & $\mathrm{~N}$ & - & - & - & - & - & + & - & $\underline{0.5}$ \\
\hline HCC8 & 6.93 & $\mathrm{H}$ & - & - & - & - & + & + & - & 1 \\
\hline HCC9 & 10.23 & $\mathrm{~N}$ & - & - & + & - & - & + & - & $\underline{2.5}$ \\
\hline HCC10 & 6.98 & $\mathrm{~N}$ & - & - & - & - & - & + & - & $\underline{0.5}$ \\
\hline HCC11 & 4.15 & $\mathrm{H}$ & - & - & + & - & - & + & - & 2.5 \\
\hline HCC12 & 9.63 & $\mathrm{~N}$ & + & - & - & + & - & + & - & $\underline{5.0}$ \\
\hline HCC13 & 26.08 & $\mathrm{H}$ & + & + & + & - & + & - & - & 8.5 \\
\hline HCC 14 & 14.09 & $\mathrm{H}$ & + & - & - & - & + & + & - & 6.5 \\
\hline HCC15 & 102.38 & $\mathrm{H}$ & + & + & + & + & + & + & + & 11.5 \\
\hline HCC16 & 6.00 & $\mathrm{H}$ & - & + & - & - & + & - & - & 2.5 \\
\hline HCC17 & 18.08 & $\mathrm{M}$ & - & - & - & - & + & + & + & 1.5 \\
\hline HCC19 & 11.46 & $\mathrm{H}$ & - & + & - & + & - & - & + & 4.5 \\
\hline HCC20 & 8.44 & M & - & + & - & - & + & - & - & 2.5 \\
\hline HCC21 & 23.10 & $\mathrm{H}$ & + & + & - & - & - & - & - & 6 \\
\hline HCC23 & 44.12 & $\mathrm{H}$ & + & + & + & + & + & + & + & 11.5 \\
\hline HCC24 & 60.03 & $\mathrm{H}$ & - & - & - & - & + & + & + & 1.5 \\
\hline HCC26 & 35.00 & $\mathrm{~L}$ & + & + & + & + & + & + & + & $\underline{11.5}$ \\
\hline HCC27 & 8.88 & $\mathrm{~N}$ & + & + & + & - & + & + & + & $\underline{9.5}$ \\
\hline HCC28 & 60.25 & $\mathrm{H}$ & + & + & - & - & - & - & + & 6.5 \\
\hline HCC29 & 5.13 & $\mathrm{H}$ & + & + & - & + & + & + & + & 9.5 \\
\hline НСС30 & 17.75 & $\mathrm{H}$ & - & + & - & + & - & + & + & 5 \\
\hline HCC29a & 164.00 & $\mathrm{~N}$ & + & + & + & + & + & + & - & $\underline{11}$ \\
\hline $\mathrm{HCC} 29 \mathrm{~b}$ & 9.03 & $\mathrm{H}$ & + & + & + & + & + & + & - & 11 \\
\hline $\mathrm{HCC} 29 \mathrm{c}$ & 275.82 & $\mathrm{H}$ & + & + & + & + & + & + & + & 11.5 \\
\hline HCC5a & 13.88 & $\mathrm{H}$ & - & + & - & + & - & - & - & 4 \\
\hline
\end{tabular}

a: Underlined numbers indicate samples with normal or low serum AFP levels.

Table 3. Patient information and CNV analysis of cfDNA samples from patients with chronic liver diseases ${ }^{a}$

\begin{tabular}{|c|c|c|c|c|c|c|c|c|c|}
\hline $\begin{array}{l}\text { patient } \\
\text { ID }\end{array}$ & age & gender & $\begin{array}{l}\text { serum } \\
\text { AFP }\end{array}$ & $\begin{array}{l}\text { serum } \\
\text { HBV } \\
\text { DNA }\end{array}$ & $\begin{array}{l}\text { plasma } \\
\text { cfDNA }\end{array}$ & $1 p-$ & $9 q-$ & $14 q-$ & $\begin{array}{l}\mathrm{CNV} \\
\text { score }\end{array}$ \\
\hline CR1 & 56 & $\mathrm{~F}$ & $\mathrm{M}$ & $\mathrm{N}$ & 25.5 & & & & 0 \\
\hline CR2 & 34 & M & $\mathrm{N}$ & $\mathrm{L}$ & 15.75 & + & & & 0.5 \\
\hline CR3 & 53 & $\mathrm{~F}$ & M & $\mathrm{H}$ & 17.63 & & & + & 0.5 \\
\hline CR4 & 59 & $\mathrm{~F}$ & $\mathrm{~L}$ & $\mathrm{H}$ & 3.27 & & + & & 0.5 \\
\hline $\mathrm{CH} 1$ & 44 & $\mathrm{~F}$ & $\mathrm{~L}$ & $\mathrm{H}$ & 16.75 & & & & 0 \\
\hline $\mathrm{CH} 2$ & 52 & M & $\mathrm{H}$ & $\mathrm{H}$ & 7.05 & & & & 0 \\
\hline $\mathrm{CH} 3$ & 33 & M & $\mathrm{L}$ & $\mathrm{H}$ & 10.45 & & + & & 0.5 \\
\hline $\mathrm{CH} 4$ & 63 & $\mathrm{M}$ & $\mathrm{N}$ & $\mathrm{H}$ & 7.35 & + & & + & 1 \\
\hline
\end{tabular}

a: see Table 1 and text for detailed description.

Table 4. Verification of NGS results by $\mathrm{PPCR}^{\mathrm{a}}$

\begin{tabular}{lllll}
\hline patient ID & $1 \mathrm{q}$ & $7 \mathrm{q}$ & $19 \mathrm{q}$ & $\begin{array}{l}\text { Internal con- } \\
\text { trol }\end{array}$ \\
\hline HCC15 & $23.11 \pm 0.80$ & $22.32 \pm 1.06$ & $23.83 \pm 0.64$ & $28.51 \pm 0.78$ \\
CR1 & $30.32 \pm 1.84$ & $26.84 \pm 1.57$ & $27.84 \pm 0.23$ & $28.51 \pm 1.03$ \\
\hline
\end{tabular}

a: Mean and SD of Ct values of 4 amplification reactions of two target regions in the same bin were shown.

\section{CNV Z score analysis}

Although in the $\mathrm{CNV}$ plots some cancer samples showed chromosome CNVs, no $\mathrm{CH}$ or $\mathrm{CR}$ samples showed recognizable changes, even some samples with tumor size $50 \mathrm{~mm}$ or less showed CNVs, most tumor samples showed no recognizable CNV changes. To look into details in the sub-cytoband level (1 M per bin), we performed CNV Z score analysis. Many potential CNV changes with $Z$ score outside -3 to +3 range could be observed in both HCC samples as well as $\mathrm{CH}$ or $\mathrm{CR}$ samples, although more extensive changes showed in HCC samples. At 1-M bin level, CNVs such as gain in $17 q, 22 q$, loss in $4 q, 13 q$ were found to be common but with less value of differential diagnosis in our sample set. It is difficult to find one significant $\mathrm{CNV}$ change in a given cytoband of a chromosome that is common in cancer samples but negative in $\mathrm{CH}$ or $\mathrm{CR}$ samples.

To focus on more specific and significant CNV changes, we counted changes meeting following criteria as positive $\mathrm{CNV}$ changes for a given chromosome arm ( $p$ or $q)$ : 1 . At least two 1-M bins in one chromosome arm with $\mathrm{Z}$ score either greater than 4.0 (gain) or less than -4.0 (loss), or 2. At least one 1-M bin in one chromosome arm with $\mathrm{Z}$ score either greater than 4.0 (gain) or less than -4.0 (loss) plus at least four other bins in the same arm with $\mathrm{Z}$ score either greater than 3.0 or less than -3.0 (same direction). With these criteria, gain in $1 \mathrm{q}, 7 \mathrm{q}, 19 \mathrm{q}$ were only found in HCC samples, while loss in $1 p, 9 q, 14 q$ were found frequently in HCC samples, but uncommon in $\mathrm{CH}$ or $\mathrm{CR}$ samples (Table 2).

To verify CNVs identified by NGS, we used fluorescence qPCR method to amplify selected regions in $1 q, 7 q, 19 q$ in two representative samples, one for HCC, one for CR. As shown in Table 4, the copy number results of the amplified regions by qPCR correlate well with CNV findings by NGS, significant gains in HCC sample with target regions having average $\mathrm{Ct}$ values 4-6 lower than the internal controls, but only minor changes in the CR sample.

\section{CNV scoring and diagnosis performance}

Gain in 1q, 7q, 19q, and loss in 1p, 9q, 14q meeting the criteria above seemingly carry some value in differential diagnosis. We then tried to score these sub-cytoband CNV changes: 2 points for each gain in $1 q$, or $7 q$, or $19 q, 0.5$ point for each loss in $1 p, 9 q$, or 14q. After comparing scores in each sample in HCC and $\mathrm{CH}, \mathrm{CR}$ groups, a threshold of 1.5 points was set for high risk for HCC (positive). In addition, a sample with any CNV that could be observed in the 150k per bin CNV plot was automatically considered as high risk for HCC, scored 4.0 points. With these scoring method, positive result (high risk for HCC) was found 
in 26 of the 31 HCC patients $(83.9 \%)$ or 11 of the 16 HCC with tumor dimension $50 \mathrm{~mm}$ or less $(68.8 \%)$ or 4 of the $7 \mathrm{HCC}$ with tumor dimension $30 \mathrm{~mm}$ or less (57.1\%), while all the 8 samples with $\mathrm{CH}$ or $\mathrm{CR}$ scored negative (Table 2, Table 3). Ten HCC patients had normal or low serum AFP levels, among them, 7 were scored positive by CNV analysis, including 4 with tumor dimension $50 \mathrm{~mm}$ or less (Table 2). The CNV scores showed poor correlation with tumor size in general (Fig. 3A). If patients were divided into three groups, with tumor size $30 \mathrm{~mm}$ or less, $31-60 \mathrm{~mm}$,

A.

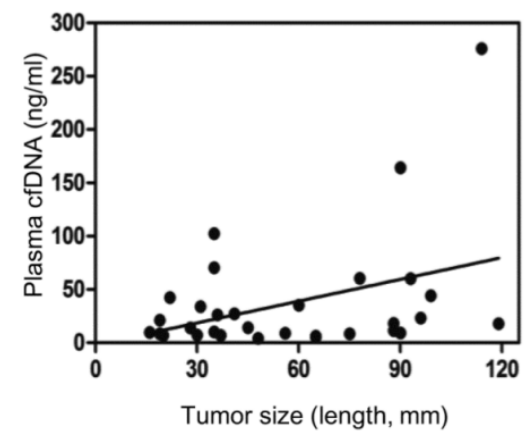

greater than $60 \mathrm{~mm}$, it was found that CNV scores steadily increased with the increase of tumor size (Fig. 3B).

It should be noted that scoring negative does not mean no CNVs, only meaning CNVs present were not sufficient to meet the criteria set here. The CNV scores were used to perform receiver operating characteristic (ROC) curve analysis using Prism 5. As shown in Figure 4 , the area under curve was 0.95 , 95\% confidence interval at $0.89-1.01$. At 1.5 cutoff point, a sensitivity of $84 \%$ and specificity of $100 \%$ were achieved. B.

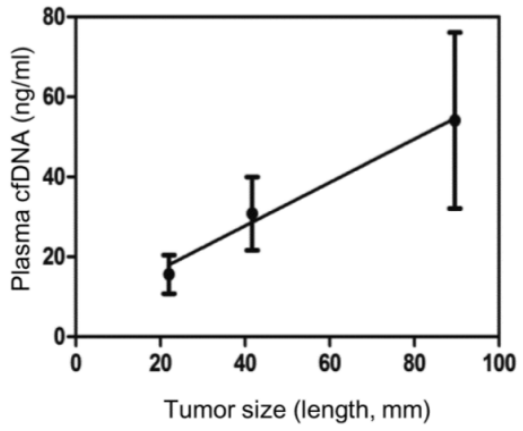

Figure 1. Correlation of plasma cfDNA concentration with tumor size. A. Scatter dot plot showing distribution cfDNA concentration and tumor size (length in $\mathrm{mm}$ ). B. Average tumor size and cfDNA concentration in three different groups based on tumor size $(<=30 \mathrm{~mm}, 31-60 \mathrm{~mm},>60 \mathrm{~mm})$, showing general trend of increase in plasma cfDNA concentration with tumor size. Dots and error bars represent Means and SD of each group.

HCC12
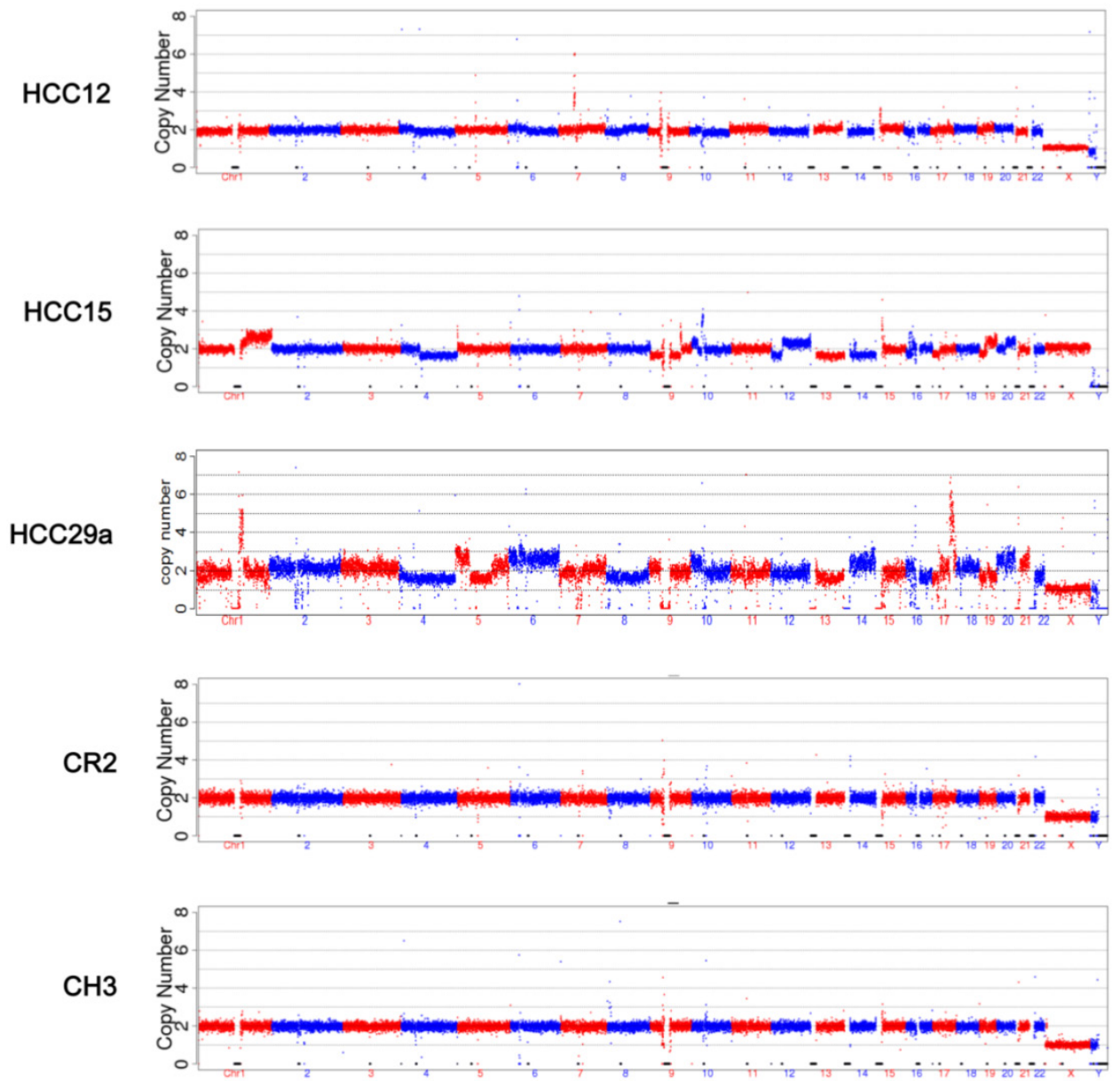

Figure 2. CNV analysis. CNV analysis was performed as described in Materials and Methods. The plots were based on data with bin size at $150 \mathrm{~kb}$. The five samples represent 5 different groups respectively: $\mathrm{HCC}$ with tumor $<=30 \mathrm{~mm}, \mathrm{HCC}$ with tumor $31-50 \mathrm{~mm}, \mathrm{HCC}$ with tumor $>50$ mm, cirrhosis, and chronic hepatitis. 
A.

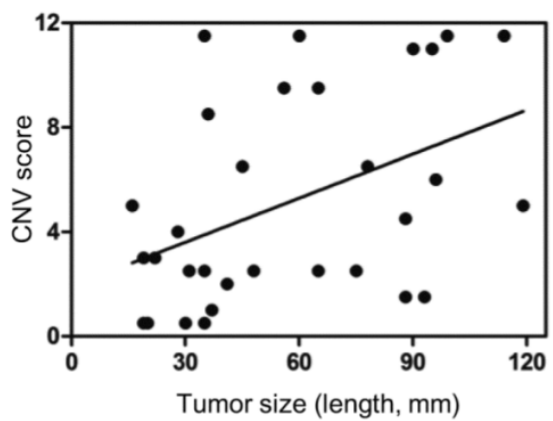

B.

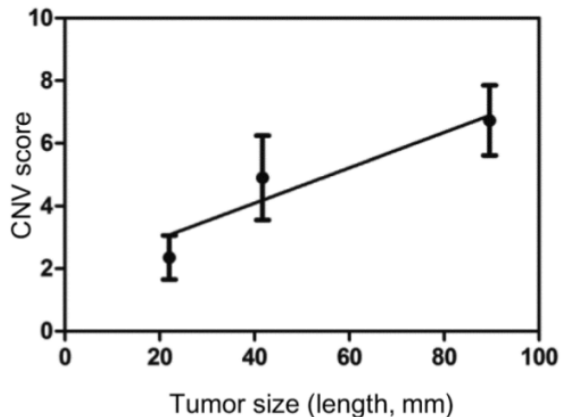

Figure 3. Correlation of CNV scores with tumor size. A. Scatter dot plot showing distribution of CNV scores and tumor size (length in mm). B. Average tumor size and CNV score in three different groups based on tumor size (<=30 mm, 31-60 mm, $>60$ mm), showing general trend of increase in CNV score with tumor size. Dots and error bars represent Means and SD of each group.

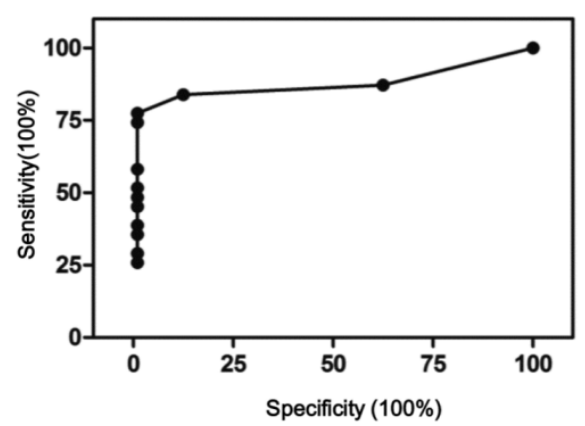

Figure 4. ROC curve of the CNV scoring method. Area under curve $0.95,95 \% \mathrm{Cl} 0.89-1.01, p<0.001$.

\section{Discussions}

Next generation DNA sequencing technology brought immense opportunities for molecular diagnosis in clinical settings. The use of non-invasive specimen, plasma cfDNA, as sources of genetic materials, coupled with NGS, offers a great option for cancer screening, early detection, treatment guiding, and disease monitoring. For an "acute cancer" such as HCC, often with significant underlying diseases as $\mathrm{CH}$ or $\mathrm{CR}$, early detection and differential diagnosis are very critical. In this pilot study, we made efforts to identify potential genomic changes by $\mathrm{CNV}$ analysis that might shed light on early detection of HCC among patients with underlying $\mathrm{CH}$ or $\mathrm{CR}$.

cfDNA quality is critical, could easily affect the test outcome. The main concern here is the lysis of patient non-tumor cells that would release large amount of non-tumor DNA into the plasma, diluting the tumor DNA present in the sample, thus dramatically reducing the test sensitivity. More controlled sample collecting and processing conditions include: using EDTA as anticoagulant, adding stabilizing agent to reduce cell lysis, complete removal of cells at plasma isolation, plasma isolation using fresh blood samples, discarding hemolytic samples, transferring plasma to $-80^{\circ} \mathrm{C}$ timely, and avoiding repeated freez- ing and thawing etc. Samples obtained under more strictly controlled conditions could offer higher quality and more reliable test results.

Equally important is the standardization of methods used in sequencing library construction, sequencing method and platform, data processing and analysis software and programs. All these could affect the test outcome significantly. Before standardized methods can be established and applied in different clinical laboratories, there would be no widely accepted diagnosis criteria or threshold.

Our results could have significant clinical implications. Currently the diagnosis of HCC mainly consists of ultrasonic or CT scan to find tumor mass (nodules) and pathology exam to verify malignancy nature and cellular origin. Many HCC developed with significant underlying liver diseases such as $\mathrm{CH}$ or CR. Often it is difficult to draw a line between the underlying disease and HCC, yet early detection of HCC is very critical for the prognosis. Serum AFP is often elevated in HCC patients, but HCC patients could have normal or low level of AFP, patients with $\mathrm{CH}$ and $\mathrm{CR}$ could have elevated serum AFP, as shown in our data. Although there are studies in protein or RNA expressions, epigenetics and microRNAs in HCC [15], fundamentally intensive genomic abnormalities are characteristics of all cancers including HCC, especially at advanced stages. The method we proposed here could identify over two thirds of HCC no more than $50 \mathrm{~mm}$, more than half of HCC no more than $30 \mathrm{~mm}$, with no positive results from $8 \mathrm{CH}$ and $\mathrm{CR}$ patients in our study. Of course, such a test only suggests high or low risk for HCC, itself alone could not be used for diagnosis. Considering it is a non-invasive method, it really has the potential to be explored and developed into a mature method that could be used in combination of imaging exams for early detection and differential diagnosis of HCC from underlying $\mathrm{CH}$ and $\mathrm{CR}$. Chan et al. performed a similar study previously with $\mathrm{CNV}$ and methylation 
exams in liver cancer. They proposed to use the percentage of bins with $\mathrm{CNV}$ and or methylation change for HCC diagnosis [9]. In our study, we found that 7 of the $8 \mathrm{CH}, \mathrm{CR}$ patients had abnormal CNV counts over 120 per sample, while 12 of 16 HCC patients with tumor size $50 \mathrm{~mm}$ or less had abnormal CNV counts less than 120 per sample, including 4 with visible CNVs in CNV plots (data not shown). Therefore, we searched for CNVs that are much less common in $\mathrm{CH}$ or $\mathrm{CR}$, but much more common in HCC, with a focus on HCC with small tumor sizes, excluding those less differential CNVs.

The study with our limited sample set showed encouraging findings. Studies with much larger samples and in different laboratories are necessary in future to verify, improve, or reshape the diagnostic application of cfDNA-NGS-CNV approach in the early detection and differential diagnosis of HCC. As stated above, scoring negative does not mean no CNVs, only meaning CNVs present were not sufficient to meet the criteria set here. The criteria for CNVs, for diagnosis threshold, the components included in the criteria, and the scoring methods could vary significantly in different laboratories and in future studies. All studies of this kind together would be able to bring mature high-tech, high throughput, highly sensitive and specific diagnostic methods to clinics that could help solve many challenging current clinical issues in patient diagnosis and treatment.

\section{Supplementary Material}

Fig.S1. http://www.jcancer.org/v06p0247s1.pdf

\section{Competing Interests}

The authors have declared that no competing interest exists.

\section{References}

1. Lim KC, Chow PK, Allen JC, et al. Systematic review of outcomes of liver resection for early hepatocellular carcinoma within the Milan criteria. Br J Surg. 2012; 99(12): 1622-1629.

2. Gullapalli RR, Lyons-Weiler $\mathrm{M}$, Petrosko $\mathrm{P}$, et al. Clinical integration of next-generation sequencing technology. Clin Lab Med. 2012; 32(4): 585-599.

3. Ong FS, Lin JC, Das K, et al. Translational utility of next-generation sequencing. Genomics. 2013; 102(3): 137-139.

4. Ulahannan D, Kovac MB, Mulholland PJ, et al. Technical and implementation issues in using next-generation sequencing of cancers in clinical practice. $\mathrm{Br}$ J Cancer. 2013; 109(4): 827-835.

5. Leary RJ, Sausen M, Kinde I, et al. Detection of chromosomal alterations in the circulation of cancer patients with whole-genome sequencing. Sci Transl Med. 2012; 4(162): 162ra154.

6. Forshew T, Murtaza M, Parkinson $C$, et al. Noninvasive identification and monitoring of cancer mutations by targeted deep sequencing of plasma DNA. Sci Transl Med. 2012; 4(136): 136ra68.

7. Shaw JA, Page K, Blighe K, et al. Genomic analysis of circulating cell-free DNA infers breast cancer dormancy. Genome Res. 2012; 22(2): 220-231.

8. Murtaza M, Dawson SJ, Tsui DW, et al. Non-invasive analysis of acquired resistance to cancer therapy by sequencing of plasma DNA. Nature. 2013; 497(7447): 108-112.

9. Chan $\mathrm{KC}$, Jiang $\mathrm{P}$, Chan $\mathrm{CW}$, et al. Noninvasive detection of cancer-associated genome-wide hypomethylation and copy number aberrations by plasma DNA bisulfite sequencing. Proc Natl Acad Sci U S A. 2013; 110(47): 18761-18768.
10. Guan XY, Fang Y, Sham JS, et al. Recurrent chromosome alterations in hepatocellular carcinoma detected by comparative genomic hybridization. Genes Chromosomes Cancer. 2000; 29(2): 110-116.

11. Zondervan PE, Wink J, Alers JC, et al. Molecular cytogenetic evaluation of virus-associated and non-viral hepatocellular carcinoma: analysis of 26 carcinomas and 12 concurrent dysplasias. J Pathol. 2000; 192(2): 207-215.

12. Wang G, Zhao $Y$, Liu X, et al. Allelic loss and gain, but not genomic instability, as the major somatic mutation in primary hepatocellular carcinoma. Genes Chromosome Cancer. 2001; 31(3): 221-227.

13. Furge KA, Dykema KJ, Ho C, et al. Comparison of array-based comparative genomic hybridization with gene expression-based regional expression biases to identify genetic abnormalities in hepatocellular carcinoma. BMC Genomics. 2005; 6: 67.

14. Zhu $\mathrm{L}, \mathrm{Hu} \mathrm{Y}$, Wang J, Zhu $\mathrm{X}$, et al. Screening for gene mutations in hypertrophic cardiomyopathy by targeted exon amplification and next generation DNA sequencing: a pilot study with three Chinese families. Exp Clin Cardiol. 2014; 20(8): 4340-4354.

15. Xing TJ, Xu HT, Yu WQ, et al. Methylation regulation of liver-specific microRNA-122 expression and its effects on the proliferation and apoptosis of hepatocellular carcinoma cells. Genet Mol Res. 2013; 12(3): 3588-3597. 